\title{
EL TRABAJO SOCIAL FORENSE Y LOS RETOS PARA SU DESARROLLO FUTURO EN PUERTO RICO
}

\section{Iván de Jesús Rosa ${ }^{1}$}

\section{Resumen}

El artículo muestra una conceptualización del trabajo social forense basándose en la literatura más reciente sobre el tema. Se señalan las áreas de intervención en el ámbito forense y las competencias necesarias para la práctica profesional. Se resalta la relevancia de diferenciar los aspectos clínicos y forenses en el ejercicio profesional. Se discuten los fundamentos metodológicos que guían la práctica según diversos modelos desarrollados. Se señalan las competencias profesionales y los aspectos legales a considerar al ejercer el rol de testigo pericial forense. Luego se discuten aspectos educativos y sobre el espacio laboral de la práctica forense en Puerto Rico. Se finaliza presentando algunos de los retos del trabajo social forense frente al contexto nacional actual.

Descriptores: trabajo social forense, metodología, testigo pericial, evaluación social.

Summary

The paper presents a conceptualization of the forensic social

${ }^{1}$ Trabajador social en la Clínica de Salud Mental de la Comunidad, Inc., adscrita a la Universidad Carlos Albizu. 
work based in the recent literature on the subject. It identifies the areas of intervention in the forensic field and the skills needed to perform professionally. It highlights the importance of differentiating between clinical and forensic practice. We discuss the methodological fundaments that guides the practice according to various models developed. It identifies the skills and legal aspects to consider when a professional is in the role of an expert witness. Then we discuss the educational issues and the work spaces of the forensic practice in Puerto Rico. It concludes with the discussion of some of the challenges of forensic social work in the current national context.

Keywords: forensic social work, methodology, expert witness, social assessment.

\section{Introducción}

Aguilar Arrieta y colaboradoras (2007, p. 3) afirman que aunque la intervención forense en trabajo social es un área de especialidad identificada recientemente "gran parte de sus actividades son tan antiguas como la profesión misma”. Otros autores como Maschi y Killian (2011) explican que la intervención forense en el trabajo social se comenzó a practicar desde el comienzo de la profesión durante la primera década del siglo XX. Aseveran que desde dicha época el trabajo social comenzó a servir en el campo legal como colaborador en el proceso de toma de determinaciones respecto a situaciones socio-judiciales. Aguilar Arrieta y colaboradoras (2007) señalan que desde aquel entonces se evaluaban a "las familias para determinar si los padres y madres estaban abusando de sus hijos/as o si no les daban respuesta a sus necesidades de desarrollo" (p. 4). Se afirma que reportaban luego sus hallazgos a las entidades de gobierno concernidas y prestaban testimonio pericial en las cortes. Estas autoras identifican a la Escuela de Trabajo Social de la Universidad de Chicago como una de las primeras en promover la inclusión de aspectos legales en el currículo de formación.

A lo largo de su desarrollo, la práctica forense del trabajo social se ha sustentado en la necesidad del poder judicial de dar solución a las situaciones de carácter psicosocial que se presentan ante dicho foro con la base de conocimientos más certera posible. Ante esto, entendemos relevante el resaltar los desarrollos más recientes sobre cómo se conceptualiza esta área de especialidad, los aspectos metodológicos en que se sustenta, el rol como testigo pericial, y el estatus actual de la educación y práctica de esta especialidad en Puerto Rico. Junto a esto, se presenta un análisis de los retos y oportunidades para el desarrollo futuro de esta área de especialidad en nuestro país.

\section{Conceptualización del trabajo social forense}

El trabajo social forense es definido por la Organización Nacional de Trabajadores Sociales Forenses de los Estados Unidos (1997) como la aplicación del trabajo social a interrogantes y asuntos relativos a la ley y al sistema judicial. Por su parte, Barrer y Branson (citados en López Beltrán, 2009), lo definen como "la práctica especializada que focaliza en la interrelación de los aspectos legales y el sistema de servicios sociales en controversias que deben ser resueltas en los tribunales” (p. 95). Mediante la utilización del término forense se hace referencia a "lo que concierne al foro, o sea, a los tribunales y a sus audiencias. Por extensión, a lo jurídico en general" (Garzón Muñoz, 2009, p. 26). 
Al abordar sus fundamentos metodológicos, Burgos Marrero (2011) define del proceso de evaluación forense como el modo "mediante el cual el/la profesional de trabajo social recopila información social, presenta y analiza los factores bio-psico-sociales que están impactando al sistema familiar; y presenta recomendaciones específicas” (p. 1). Por su parte, Ovares Pacheco, González Aguilar y González Brenes (2007), articulan el peritaje social forense como "una forma de evaluación compleja, donde lo que se perita no es un objeto estático, sino sujetos activos del derecho: las personas y su dignidad" (p. 3). Resaltan que la evaluación forense "contiene una operación valorativa, sustentada en consideraciones técnicas de la disciplina, pero trasciende la simple narración de resultados y percepciones, y conlleva una opinión profesional en torno a la realidad investigada" (p. 3).

Garzón Muñoz (2009) asevera que en el campo forense los y las profesionales del trabajo social "tienen una labor de apoyo y asesoramiento al juez y/o al fiscal, a través del peritaje social” (p. 28). Para esto, deben realizar una investigación al núcleo familiar a ser evaluado y los recursos con lo que ésta cuenta. Para luego emitir las conclusiones y recomendaciones que se entiendan pertinentes.

Por otra parte, Faller (2007) describe la práctica forense diferenciándola de la práctica clínica. Establece que en el campo forense quien recibe los servicios de trabajo social lo es la corte, a diferencia del ámbito clínico donde lo es la niñez y sus familias. Indica que la intervención forense se da dentro de un contexto legal, mientras que la clínica parte de un contexto terapéutico. Afirma que el posicionamiento del o la profesional forense debe ser de objetividad frente a la situación evaluada, por el contrario el o la profesional clínico desarrolla una relación de sostén y apoyo hacia su cliente. La información que se recopila en el ámbito forense debe basarse sólo en hechos concretos, mientras que en el ámbito clínico la información se basa en las experiencias subjetivas de las personas involucradas. Asevera la autora que toda intervención forense debe estar fundamentada estrictamente en protocolos apoyados por la comunidad profesional.

Profesionales en el ámbito clínico pueden ser más flexibles, considerando las necesidades particulares de sus clientes. Otro contraste es que la evaluación forense debe contar con diversos contactos a colaterales pertinentes a la situación con el objetivo de triangular la información. En la intervención clínica no es indispensable hacer contacto con colaterales. Finalmente, diferencia el tipo de informe a ser radicado luego de la intervención profesional, el forense debe ser uno largo y detallado donde se ofrezcan de manera explícita todos los fundamentos que llevan a la conclusión profesional. Los informes de terapia clínica tienden a ser cortos, exponiendo el funcionamiento general de la persona y proveyendo algún tipo de diagnóstico.

Tomando en consideración los contrastes antes señalados, Heilbrun (2001) resalta la importancia de comenzar un proceso de evaluación forense notificando a las personas a ser evaluadas sobre la naturaleza y los objetivos específicos de la evaluación. Esto, haciendo especial énfasis en que la información que brinden será notificada en su totalidad al sistema judicial. Por lo cual, no se debe tener la expectativa de que se utilizarán los mismos estándares de confidencialidad aplicados a la intervención clínica.

VOCES DESDE EL TRABAJO SOCIAL

NúM.1 
En el contexto forense destaca el que el o la profesional del trabajo social debe ejercer exclusivamente una función evaluadora. Esto, con el objetivo de orientar al tribunal de manera objetiva sobre el asunto evaluado (Córdova Campos, 2010). El que un mismo profesional ofrezca servicios de evaluación a un núcleo familiar al que haya atendido terapéuticamente con anterioridad se considera altamente inadecuado, ya que generaría una dualidad de roles incompatibles entre sí (Kuehnle, 1996). Esta situación podría levantar cuestionamientos éticos sobre la objetividad del evaluador o evaluadora al momento de su intervención.

El dominio avanzado que deben tener profesionales del trabajo social forense exige la especialización en un área específica del conocimiento de la realidad social. Por lo cual, deben reconocer y establecer los límites de su área de peritaje. Esto implica no aceptar realizar evaluaciones forenses en áreas en las cuales no tengan un conocimiento especializado, y a su vez un esfuerzo educativo constante con el objetivo de mantener actualizado el conocimiento científico pertinente a su área de intervención.

Las áreas de peritaje en la práctica del trabajo social son múltiples. Las mismas pueden abordar ámbitos tan diversos como la salud mental, la educación especial y la adopción, entre muchas otras. Lo que determinará la competencia para poder servir como especialista en dichas áreas lo será principalmente la educación y la experiencia en el ámbito de peritaje.

Figura \# 1

Ejemplo de áreas de especialidad en la práctica del trabajo social forense

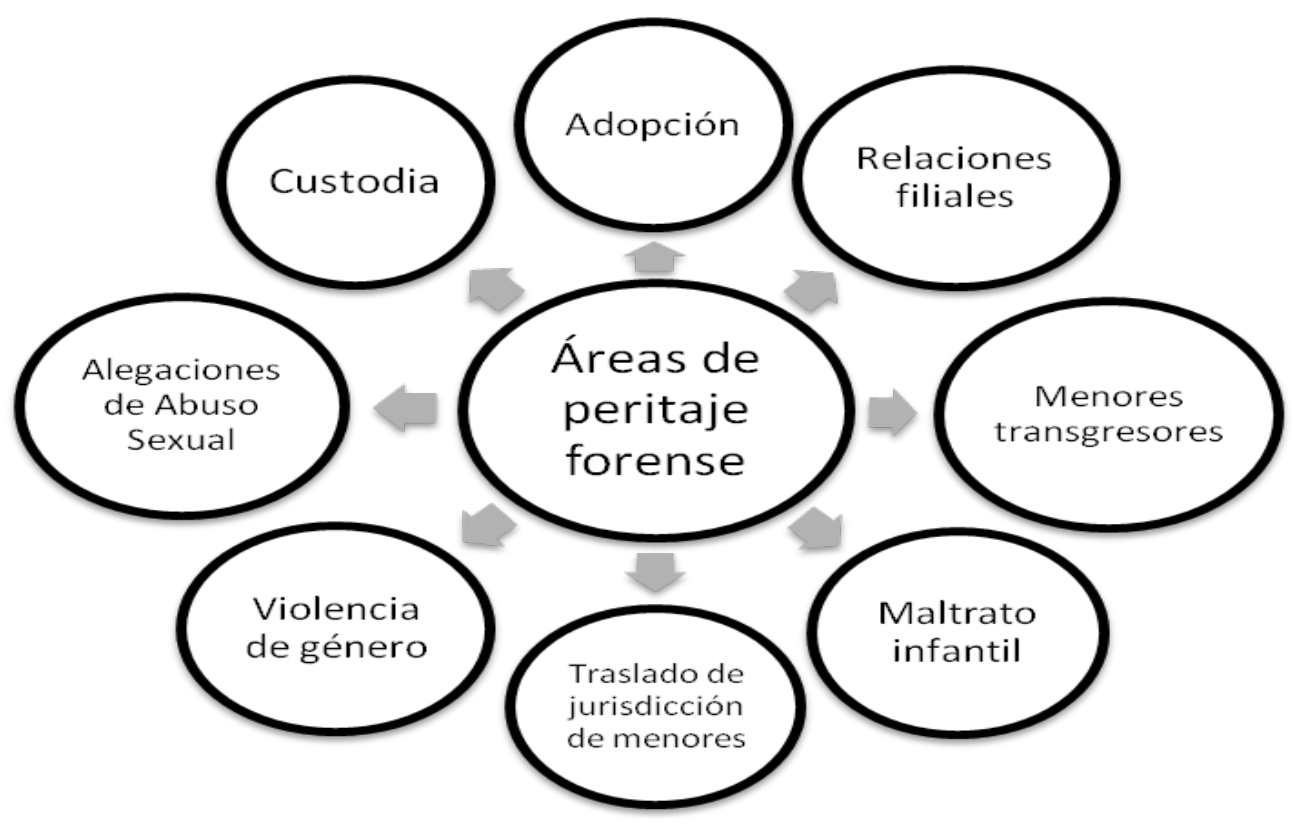

Fundamentos metodológicos de la práctica forense

Ovares Pacheco y colaboradoras (2007) afirman que la práctica forense en trabajo social ha logrado el desarrollo teórico y metodológico suficiente como para sostenerse como un modelo de intervención de la práctica. Conceptualizan dicho modelo como la "estructura metodológica que... se utiliza para la intervención y/o el abordaje de las diferentes situaciones o problemas individuales, familiares y grupales que afectan a la sociedad y que trascienden a la esfera judicial" (p. 3).

Por otra parte, López Beltrán (2009) sostiene que el proceso de evaluación forense parte de una base empírica que se articula

VOCES DESDE EL TRABAJO SOCIAL

NÚM.1 
mediante el método científico. Apunta que la evaluación debe descansar en la observación y la medición para llegar a conclusiones apoyadas por la evidencia. Para Ander-Egg (1984) el empirismo es entendido como la doctrina filosófica que señala que el verdadero conocimiento es sólo aquel que se deriva de la experiencia. Añade que "lo propio de la práctica científica que se deriva de esta concepción, es el recoger datos, comprobarlos, remitirlos, sistematizarlos y analizarlos por un proceso de abstracción" (p. 110).

Basándose en dicho paradigma, López Beltrán (2009) señala que los pasos del proceso de evaluación forense son:

a) se define el problema-motivo del referido... b) se revisa la literatura y el marco teórico a la luz de la conducta observada... c) se formulan hipótesis para explicar la posible motivación de la conducta... d) se diseña la metodología-protocolo de evaluación... e) se recopilan los datos... f) se analizan los datos, y g) se emiten conclusiones y se hacen recomendaciones (p. 106-107)

Ovares Pacheco y colaboradoras (2007), aún reconociendo el carácter empirista del proceso evaluativo, señalan que "la investigación cualitativa se ha constituido en uno de los pilares para construir los procesos de investigación que se realizan" (p. 17). Aguilar Arrieta y Briceño Castro (2007) también sostienen que la intervención forense "parte de una base metodológica cualitativa, la cual procura la transformación de los datos en interpretaciones sustentadas científicamente por diversas teorías" (p. 5). Afirman que las finalidades del proceso evaluativo, enmarcado dentro del paradigma cualitativo, son: “a) la búsqueda del significado de los fenómenos a partir de datos concretos, b) confirmar o rechazar hipótesis, y c) ampliar la comprensión de la realidad como una totalidad" (p. 5). Estipulan que, dentro del paradigma cualitativo, la perspectiva investigativa que más se acerca al quehacer forense lo es la hermenéutica. Por lo cual, se busca interpretar el comportamiento humano desde el propio marco de referencia de las personas investigadas y desde el contexto en que el mismo se da (Lucca Irizarry y Berrios Rivera, 2009).

Entre las estrategias cualitativas para obtener información pertinente al proceso de evaluación forense se destacan la entrevista a profundidad, la observación no partícipe y el análisis de documentos. Bajo el paradigma cuantitativo resalta el uso de pruebas proyectivas con propiedades sicométricas, de cuestionarios y de pruebas estandarizadas (Faller, 2007).

A continuación, se presenta el marco metodológico de la labor pericial forense utilizado por profesionales del trabajo social del Departamento de Trabajo Social y Psicología del Poder Judicial de Costa Rica. Este modelo de intervención fue establecido por colegas en el Poder Judicial Costarricense para guiar su intervención profesional. El presente es un resumen de lo presentado por Aguilar Arrieta y colaboradoras (2007). Para una exposición detallada de este modelo, se les refiere a dicha fuente.

\section{Acercamiento inicial a la situación objeto de evaluación}

Se explica que mediante el análisis de contenido se realiza 
una exploración previa al contacto inicial. Afirman que mediante la misma se exploran los antecedentes del caso a través de la revisión de documentos. Mediante esta estrategia se determina la documentación pertinente al caso. Exponen que se auscultan las intervenciones previas de otras instituciones en el núcleo familiar antes del conflicto judicial. Manifiestan que de esta forma se define inicialmente el problema a evaluar y los objetivos de ésta. Estipulan que desde el acercamiento inicial se debe comenzar a revisar literatura y establecer el marco teórico a utilizarse considerando el asunto a evaluar.

\section{Evaluación preliminar con las partes}

Explican que en este momento se procede con la realización de las entrevistas forenses. Señalan que se entrevista a todas las partes involucradas en el proceso evaluativo y a otras fuentes de información colateral. Afirman que las entrevistas pueden ser en una o varias sesiones. Esto, dependiendo de la situación-problema evaluado y del protocolo de intervención establecido. También enfatizan en esta etapa el uso de la técnica de observación, señalando que viabiliza el "consignar todas las percepciones respecto a las manifestaciones conductuales, actitudes y afectos visibles que la persona por evaluar pone de manifiesto durante el proceso de investigación” (p. 47). Resaltan que el uso de esta técnica también permite integrar al proceso evaluativo el contexto ambiental de la familia evaluada.

\section{Fase interpretativa o analítica y planteamiento de hipótesis}

Se explica que luego de recopilar la información mediante las estrategias previamente discutidas se procede con la elaboración de diversas hipótesis que puedan brindar posibles explicaciones a las preguntas planteadas como parte de la evaluación. Se busca identificar cuáles pueden ser las causas que expliquen la situación evaluada. Afirman que esta tarea se va perfeccionando durante el progreso de las próximas etapas de la investigación.

\section{Fase de planificación, acceso a otras fuentes de información y estudio de campo}

Señalan que en este momento se deben determinar las técnicas y estrategias a seguir en el resto del proceso. Indican que se deben contestar las preguntas “¿Qué voy a realizar? ¿Cómo lo voy a hacer? ¿Dónde? ¿Con qué lo voy a hacer?” (p. 44). Destacan que entre las estrategias de recopilación de información en esta etapa se encuentran la visita a otros familiares, escuelas, hospitales, la comunidad y al servicio de bienestar infantil.

\section{Análisis, integración de resultados y elaboración del peritaje social}

Dentro de este modelo, se plantea como paso final el análisis de toda la información recopilada. Se indica que el análisis debe realizarse mediante la sistematización de los datos recopilados. Esto, de manera objetiva y científica. Afirman que se deben destacar todas las variables evaluadas, señalando sólo lo esencial del caso. Por último, se emiten las conclusiones y se brindan las recomendaciones que se entiendan pertinentes.

Subsiguientemente, se presenta la estructura de evaluación forense propuesta por Heilbrun (2001). La lectura de la misma 
permite comparar y contrastar las fases identificadas en los modelos antes expuestos. Se debe recordar que cada autor o autora determinará qué aspectos resaltar y cuáles no. Sin embargo, todo modelo debe ser congruente con el método científico y el abordaje empirista a la realidad social.

\section{Figura \# 2}

Estructura organizativa del proceso de evaluación forense según (Heilbrun, 2001)

\begin{tabular}{|c|c|c|}
\hline \multicolumn{3}{|c|}{ Preparación de la intervención } \\
\hline Contactos iniciales & Definición del rol del evaluador & Selección del modelo \\
\hline \multicolumn{3}{|c|}{ Recopilación de los datos } \\
\hline Selección de las fuentes de información & Ejecución de las técnicas de recopilación \\
\hline \multicolumn{3}{|c|}{ Interpretación de los datos } \\
\hline \begin{tabular}{|c|c|c|}
\hline \multicolumn{3}{|c|}{ Incorporación del razonamiento } \\
científico a la información
\end{tabular} & $\begin{array}{c}\text { Realizar afirmaciones y clarificar } \\
\text { los límites de la evaluación }\end{array}$ \\
\hline \multicolumn{3}{|c|}{ Co la información } \\
\hline \multicolumn{3}{|c|}{ Comunicación } \\
\hline
\end{tabular}

Es importante destacar que los modelos anteriores buscan resaltar lo que deben ser los fundamentos de toda intervención forense. Sin embargo, esto no sustituye el uso de las guías y protocolos de intervención que deben ser utilizados basándose en el área de la realidad social que se desea evaluar (violencia de género, custodia, etc.). Cada una de estas áreas de intervención cuenta con guías y protocolos establecidos por organizaciones profesionales que atienden dichos temas. El uso de estas guías se considera indispensable, ya que mediante el uso de las mismas se logra abordar las particularidades que cada tipo de evaluación forense demanda y se le añade fiabilidad al proceso evaluativo.

\section{El trabajador/a social forense como testigo pericial}

Como trabajadores y trabajadoras sociales forenses el objetivo final de la intervención profesional será servir como testigo pericial en los procesos judiciales relacionados con la situación analizada. El rol como testigo pericial está detalladamente regulado por las Reglas de Evidencia de Puerto Rico del año 2009, las cuales establecen el proceso mediante el cual se maneja todo tipo de evidencia en las salas del Tribunal General de Justicia de Puerto Rico.

Según la regla 703, para que una persona pueda ser considerada como perita, ésta debe poseer "especial conocimiento destreza, experiencia, adiestramiento o instrucción suficiente para calificarla como experta o perita en el asunto sobre el cual habrá de prestar testimonio" (p. 46). Según la regla 702, luego se ser cualificado como perita, el valor probatorio del testimonio dependerá de:

(a) si el testimonio está basado en hechos o información suficiente; (b) si el testimonio es el producto de principios y métodos confiables; (c) si la persona testigo aplicó los principios y métodos de manera confiable a los hechos del caso; (d) si el principio subyacente al testimonio ha sido aceptado

VOCES DESDE EL TRABAJO SOCIAL

NÚM.1 
generalmente en la comunidad científica; (e) las calificaciones o credenciales de la persona testigo; y

(f) la parcialidad de la persona testigo.

La trabajadora o trabajador social forense deberá tener la capacidad de defender su evaluación considerando los puntos antes señalados. Esta normativa demanda adiestramiento constante y un alto dominio de su área de especialidad. Dicho conocimiento le deberá permitir defender de manera exitosa el proceso evaluativo que realizara. Profesionales forenses carentes del dominio teórico y metodológico para defender su análisis en el tribunal, pueden correr el grave riesgo de quedar desacreditados o desacreditadas ante dicho foro, y de que se intente impugnar su evaluación. Por lo tanto, no basta con que el trabajador o trabajadora social tenga estudios especializados en la disciplina. Si no, se debe demostrar que se domina a plenitud el área de conocimientos de la evaluación que realizara (maltrato infantil, relaciones filiales, etc.). También debe asegurarse de haber guiado su proceso evaluativo utilizando las guías y estándares concertados por la comunidad profesional a la cual pertenezca.

La Sociedad Profesional Americana en el Abuso Infantil (1997) ha establecido las características que debe tener un evaluador o evaluadora forense en el área de abuso sexual. Dichas características se pueden extrapolar a los y las evaluadoras forenses que se especialicen en otras áreas de intervención. Estas características son: a) poseer un grado académico graduado en el área de conducta humana (en el caso del trabajo social: M.S.W.,
D.S.W. o Ph.D.). b) Tener experiencia profesional en la intervención con familias y en su área de peritaje. Se recomienda un mínimo de tres años de experiencia, de no poseerla, la supervisión se considera indispensable. c) Tener entrenamiento especializado en el área de peritaje. El adiestramiento se debe evidenciar mediante cursos formales, supervisión, asistencia a conferencias, seminarios y talleres. d) Estar familiarizado con la literatura y los asuntos relevantes a su área de peritaje. f) Conocer los diversos valores culturales y las prácticas familiares que puedan influir en la situación a evaluar. g) Tener experiencia conduciendo evaluaciones forenses y proveyendo testimonio pericial. Por último se estipula que, h) se debe acercar a la evaluación con la mente abierta, considerando todas las posibles explicaciones y considerando hipótesis alternas.

Según López Beltrán (2009) otras destrezas que debe poseer un trabajador o trabajadora social forense son: conocimiento de la legislación y jurisprudencia relativa al caso bajo análisis, la estructura y el funcionamiento de la rama judicial, y el dominio de los hallazgos científicos actualizados y de las teorías pertinentes a la evaluación. Adicionalmente, Rodríguez Olmo (2011) añade que, ante todo, se debe tener el compromiso ético de siempre decir la verdad ante el tribunal.

Ceci y Hembrooke (1998) resaltan los diversos dilemas éticos que pueden surgir cuando un perito o perita forense recibe pago por sólo una de las partes en conflicto. Afirman que aun así se espera que el perito ofrezca un testimonio verdaderamente objetivo, sin importar la parte que page sus honorarios. Reconociendo esto, consideran como una rara excepción el que un experto o experta
Según Lóp social forer análisis, la gos científi Rodríguez siempre de 
esté en disposición de favorecer mediante un testimonio fraudulento a una de las partes por razones económicas. Afirman que la gran mayoría de los peritos y peritas se esfuerzan por ofrecer un testimonio balanceado y por evitar conflictos de interés, tanto reales como en apariencia.

No hacerlo así implicaría una crasa violación a los cánones de ética establecidos por nuestra profesión. Estos cánones, al abordar los aspectos relacionados a la integridad en nuestra intervención profesional, indican que se debe identificar y enfrentar "las posibles influencias y presiones ajenas a su función que puedan interferir con el ejercicio de su discreción y su juicio imparcial" (Colegio de Profesionales del Trabajo Social de Puerto Rico, 2011, p. 24).

\section{El trabajo social forense en Puerto Rico}

López Beltrán (2009) reseña que la presencia de trabajadores y trabajadoras sociales en los tribunales de justicia del país se remonta al año 1947, momento en que se comenzaron a contratar profesionales del trabajo social para asesorar a los jueces y juezas en el área de relaciones de familia. La autora indica que en el año 1954 se financió un estudio con el propósito de establecer la necesidad de los servicios sociales en los tribunales. Dicho estudio viabilizó la creación de "la estructura administrativa para la implantación de los servicios sociales en todas las Salas del Tribunal" (p. 83). López Beltrán (2009) presenta un recuento de las transformaciones principales de los ofrecimientos de servicios sociales en la judicatura hasta establecerse en el año 2003 la Oficina de Servicios Sociales (OSSO), bajo la Directoría de Programas Judiciales.

Destaca el hecho que en el contexto puertorriqueño la práctica del trabajo social forense se ha llevado a cabo principalmente por profesionales con destaque en esta oficina de la Rama Judicial. La Oficina de Servicios Sociales articula como su misión el "garantizar que los servicios sociales de apoyo pericial a los jueces y las juezas se brinden de acuerdo con la política pública, los parámetros establecidos por la profesión, y la normativa establecida por legislación o por la Rama Judicial”' (OSSO, s/f, párr. 1).

En la Oficina de Servicios Sociales, profesionales del trabajo social realizan evaluaciones forenses en el área de custodia, relaciones filiales, menores transgresores y de traslado de menores hacia otra jurisdicción política. Mientras, colegas empleados y empleadas por el Departamento de la Familia realizan como parte de su labor ordinaria evaluaciones forenses en el área de maltrato a menores y adopción, amparándose en la Ley para el Bienestar y la Protección Integral de la Niñez del año 2003. Asimismo, organizaciones que se especializan en el área de violencia por género y abuso sexual infanto-juvenil contratan los servicios de profesionales del trabajo social con peritaje para realizar evaluaciones forenses en dichas áreas de conocimiento (González Torres, 2001). Cabe resaltar que en Puerto Rico se cuenta con múltiples colegas que ejercen la práctica forense de manera independiente mediante contratos de servicios profesionales otorgados por personas $\mathrm{u}$ organizaciones interesadas en su peritaje.

Munson (2011) afirma que el análisis profesional en el ámbito forense debe partir de la unión de los conocimientos en el área de conducta humana y el área legal, ya que se requiere una comprensión sustancial, no sólo de los problemas psicosociales que 
afectan al núcleo familiar evaluado, sino de las implicaciones que el espacio jurídico tendrá en dicha familia. Es por esto que critica el hecho de que el conocimiento en el campo legal regularmente no se obtiene mediante la educación medular ofrecida en la disciplina de trabajo social. Postula que dichos conocimientos tienden a obtenerse mediante programas de adiestramiento especializado como profesional forense y testigo pericial. Concluye que sin una preparación formal apropiada no se podría ejercer con destreza los roles antes señalados.

En Puerto Rico, la educación en el área de trabajo social forense es relativamente reciente. Los primeros esfuerzos identificados en la literatura (López Beltrán, 2003) provinieron de la Oficina de Servicios Sociales de la Rama Judicial. Desde dicha oficina se instauraron las Conferencias de Trabajo Social Forense, las cuales se realizan desde el año 1999 bienalmente. Ya con siete conferencias realizadas, este evento se ha establecido como uno de los espacios de mayor prominencia en el proceso de educación continuada en trabajo social en el país. En estas conferencias se discuten diversos temas pertinentes al proceso de evaluación forense. Las conferencias han estado dirigidas principalmente a los trabajadores y trabajadoras sociales que laboran en la Rama Judicial, aunque profesionales de otros espacios laborales también se han podido beneficiar de este ofrecimiento educativo. Las mismas se han realizado con la colaboración de diversas universidades, del Colegio de Profesionales del Trabajo Social de Puerto Rico y de otras organizaciones profesionales.
Durante el año 2011 se dio un gran paso de avance en la educación en el área forense. Dicho año se llevó a cabo el primer ofrecimiento académico en Puerto Rico dirigido a alcanzar una formación universitaria en trabajo social forense. La misma, ofrecida por la Universidad del Turabo, estuvo dirigida a la obtención de una certificación profesional posgraduada en trabajo social forense. Esto, mediante un adiestramiento de siete meses de duración, para un total de ciento cincuenta y seis horas contacto. Dicha iniciativa fue establecida con el apoyo del Instituto de Educación Continuada del Colegio de Profesionales del Trabajo Social. La concretización de dicho programa resultó en un amplio avance en el proceso educativo en trabajo social en el país. Aunque ciertamente aún falta mucho por alcanzar.

\section{Retos para la práctica forense en el Puerto Rico actual}

Al momento de redacción de este artículo la nación puertorriqueña atraviesa por uno de los momentos más críticos de su historia. Colón Reyes, desde el año 2007, advertía sobre esta crisis indicando que "socialmente el desgaste de las esferas económicas se deja sentir en los altos niveles de desempleo, la criminalidad, la deserción escolar, la corrupción y la anomia reinantes" (p.92). Frente a este panorama Gutiérrez (citado en Rivera Vargas, 2011) expone, al considerar el impacto de la oleada de las políticas neoliberales impulsadas por la presente administración de gobierno, que éstas "han generado aumentos de violencia en todas las partes donde se han implantado" (párr. 10).

En dicha noción ideológica es que se han fundamentado nuestros actuales dirigentes gubernamentales para realizar enormes recortes en servicios públicos y a programas dirigidos al área de 
bienestar social. Se destacan el despido masivo de empleados de gobierno, la eliminación del Programa de Comunidades Especiales, el aumento en los costos de la educación universitaria pública, entre otras iniciativas (Aponte Ortiz, 2009; Cotto, 2009).

Mientras, se perfila que el año 2011 será el más violento de nuestra historia. De continuar el patrón actual, se espera que antes de culminar el año se establezca un récord en la cifra de asesinatos, sobrepasando las mil muertes violentas. Alcanzando también una cifra record en las muertes por violencia de género (Caro, Rodríguez y Cobián, 2011; Rivera Vargas, 2011). Por otra parte, los casos atendidos por el Departamento de la Familia (D.F.) relacionados con alegaciones de abuso sexual se estiman en unos 2,000 al año. (D.F., 2007). Bajo este panorama, se hace necesario un mayor nivel de desarrollo por parte de los programas concernidos a evaluar desde el ámbito forense las problemáticas sociales antes planteadas.

Considerando el excelente trabajo realizado por colegas que laboran en las Unidades Sociales de Familia y Menores de la Oficina de Servicios Sociales de la Rama Judicial de Puerto Rico, se debería ponderar la ampliación de los servicios ofrecidos por dicha unidad. Se podría utilizar como modelo los avances alcanzados por el Departamento de Trabajo Social y Psicología del Poder Judicial de Costa Rica. Allí, además de contar con los Programas Penal Juvenil y de Familia (equivalentes a los existentes en Puerto Rico), se han desarrollado los programas de Atención a la Violencia Sexual InfantoJuvenil y el de Violencia Doméstica.

Por ejemplo, el Programa de Atención a la Violencia Sexual Infanto-Juvenil se plantea como objetivo el "desarrollar procesos de valoración social orientados a analizar las condiciones familiares, sociales, ambientales y personales de la población referida y, de forma específica, intervinientes en la situación de violencia sexual investigada" (Aguilar Arrieta y colaboradoras, 2007, p. 110). Señalan además, que el programa busca atender a menores de edad ( 2 a 18 años) y a sus familiares, en situaciones en las cuales se haya radicado alguna denuncia por delitos sexuales.

Se resalta que entre los servicios que ofrece dicho programa se encuentra la investigación social. Mediante ésta, se realiza una evaluación forense comprensiva del menor y su núcleo familiar en relación a la alegación de abuso denunciada. Se identifican las consecuencias asociadas o derivadas de la alegación de abuso y se finaliza con el producto de un peritaje social donde se presentan los hallazgos y análisis de la información. Destacan también el servicio de atención individual y grupal mediante un acercamiento socioeducativo. Mediante este enfoque se proponen abordar las “etapas y características del proceso judicial, derechos de la persona menor de edad en el proceso y familiarización con sala de juicio; sistema de apoyo (personas de confianza); y el plan de seguridad y prevención a nivel familiar y personal" (Aguilar Arrieta y colaboradoras, 2007, p.115).

El incorporar iniciativas como esta a los servicios sociales ofrecidos desde la judicatura, no sólo exponenciaría los ofrecimientos de dicha oficina, sino que ayudaría a palear los vacíos dejados por otros programas. Esto, ante el recorte en las asignaciones fiscales de la rama ejecutiva a los programas que ofrecen este tipo de servicios desde el tercer sector.

También recomendaría un mayor abordaje de las áreas de peritaje social forense desde los ambientes formativos a nivel de

VOCES DESDE EL TRABAJO SOCIAL

NúM.1 
bachillerato y maestría en trabajo social. Las especificidades que este campo de intervención exige, demanda de la academia un mayor nivel de enfoque en las áreas temáticas que capacitan a colegas en su función evaluadora y como testigo pericial en el ámbito judicial. Iniciativas educativas como las descritas con anterioridad deberían ser ampliadas y emuladas.

Por último, reconociendo el trabajo que se ha venido realizando desde la institucionalidad de la Rama Judicial, plantearía la posibilidad de la creación de una organización compuesta por colegas forenses, ya sea dentro o fuera de nuestro colegio profesional. Esto, con el objetivo de que se puedan crear espacios de discusión donde se explore el estatus actual de esta especialización en Puerto Rico y se fomenten estrategias para su desarrollo futuro. Mediante una iniciativa de esta naturaleza se podrían establecer proyectos para ampliar espacios educativos, originar literatura profesional, fomentar cambios en la política social vigente, y promover la integración de más profesionales a la práctica independiente en el campo forense, entre otros retos que se podrían asumir desde esta área de especialidad.

\section{Referencias}

Aponte Ortiz, Félix I. (2009, 20 al 26 de agosto). La tormenta perfecta ante un gobierno inepto. Claridad, p. 8

Aguilar Arrieta, Ivette y Briceño Castro, Ana S. (2007, septiembre). La intervención de trabajo social forense en la atención de personas menores de edad que han denunciado delitos sexuales, en el Primer Circuito Judicial de San José. Poder Judicial Costarricense. Ponencia presentada en el IV Congreso Internacional - VII Congreso Nacional de Trabajo Social en San José, Costa Rica.

Aguilar Arrieta, Ivette; Chacón Mora, Laura; González Aguilar, Matilde; González Brenes, Rosario; Jiménez Villalobos, Sheiris; Loaiza Coronado, Mercedes; et al. (2007). La intervención de trabajo social y psicología en la administración de justicia costarricense. Costa Rica: Editorama.

Ander-Egg, Ezequiel. (1984). Diccionario del trabajo social. México: Editorial El Ateneo.

Burgos Marrero, Sylvia L. (2011, febrero). La evaluación social forense. Adiestramiento presentado como parte de la Certificación en Trabajo Social Forense en la Universidad del Turado en Gurabo, Puerto Rico.

Caro, Leysa; Rodríguez, Arys y Cobián, Mariana. (2011, 7 de octubre). Sin tregua la violencia doméstica. Primera Hora, p. 6 
Ceci, Stephen J. y Hembrooke, Helene. (Ed.). (1998). Expert Witnesses in Child Abuse Cases. Estados Unidos: American Psychological Association.

Colegio de Profesionales del Trabajo Social de Puerto Rico. (2011). Código de ética profesional. Puerto Rico: Autor.

Colón Reyes, Linda. (2007). La tarea inconclusa: pobreza y desigualdad social en el siglo XXI. Revista de Ciencias Sociales, 17, 78-117.

Córdova Campos, Rita. (2010). Trabajo social clínico en Puerto Rico: Construcción de la personalidad puertorriqueña. Puerto Rico: Action Printing.

Cotto, Cándida. (2009, 12 al 18 de noviembre). Gobierno de Fortuño desconoce pobreza y desigualdad. Claridad, p. 6

Departamento de la Familia. (2007). Plan nacional para la prevención de maltrato a menores. Puerto Rico: Autor.

Faller, Kathleen. (2007). Interviewing Children About Sexual Abuse: Controversies and Best Practice. Estados Unidos: Oxford University Press.

Garzón Muñoz, Rubén D. (2009). Trabajo social forense y maltrato infantil. Colombia: Anzuelo Ético.

VOCES DESDE EL TRABAJO SOCIAL
González Torres, Doris. (2001, mayo). Proyecto Amanecer. Ponencia presentada en la Segunda Conferencia de Trabajo Social Forense en la Universidad del Sagrado Corazón. San Juan, Puerto Rico.

Heilbrun, Kirk. (2001). Principles of Forensic Mental Health Assessment. Estados Unidos: Kluwer Academic / Plenum Publishers.

Kuehnle, K. F. (1996). Assessing Allegations of Child Sexual Abuse. Estados Unidos: Professional

Resource Press.

Ley para el Bienestar y la Protección Integral de la Niñez, Ley 177. Agosto, 03. P. del S. 2285.

López Beltrán, Ana M. (2003, junio). Introducción y propósito. Ponencia presentada en la Tercera Conferencia de Trabajo Social Forense en la Universidad Interamericana. San Juan, Puerto Rico.

López Beltrán, Ana M. (2009). La práctica especializada en trabajo social forense. Puerto Rico: BiblioGráficas.

Lucca Irrizari, Nydia y Berrios Rivera, Reynaldo. (2009). La investigación cualitativa: fundamentos, diseños y estrategias. Puerto Rico: Ediciones SM.

VOCES DESDE EL TRABAJO SOCIAL 
$\overline{\text { Maschi, Tina y Killian, Mary L. (2011). The Evolution of Forensic }}$ Social Work in the United States: Implications for 21st Century Practice. Journal of Forensic Social Work, 1:1, 8 - 36.

Munson, Carlton. (2011). Forensic Social Work Practice Standards: Definition and Specification. Journal of Forensic Social Work, $1: 1,37-60$.

National Organization of Forensic Social Work. (1997). What is Forensic Social Work?. Tomado el 5 de septiembre de 2011 de http://www.nofsw.org/html/forensic_social_work.ttml.

Ovares Pacheco, Érika; González Aguilar, Matilde y González Brenes, Rosario. (2007, septiembre). El trabajo social forense como modelo de intervención: La experiencia en el Poder Judicial, Costa Rica. Ponencia presentada en el IV Congreso Internacional - VII Congreso Nacional de Trabajo Social en San José, Costa Rica.

Oficina de Servicios Sociales. (s/f). Misión y logros. Recuperado el 1 de septiembre de 2011 de http://www.ramajudicial.pr/DPJ/ Oficina-de-Servicios-Sociales-(OSSO).htm.

Rivera Vargas, Daniel (2011, 6 de septiembre). El crimen no para. El Nuevo Día, p. 3

Rodríguez Olmo, Rafael. (2011, junio). Testimonio pericial: Rol del trabajador social como testigo pericial. Adiestramiento presentado como parte de la Certificación en Trabajo Social Forense en la Universidad del Turado en Gurabo, Puerto Rico.
Sociedad Profesional Americana en el Abuso Infantil. (1997). Guidelines for psychosocial evaluation of suspected sexual abuse in children. Chicago: Autor.

Tribunal Supremo de Puerto Rico. (2009). Reglas de evidencia de Puerto Rico. Puerto Rico: Autor. 